\title{
DIVERSIDADE DE ESPÉCIES E VARIEDADES DE SEMENTES CRIOULAS NOS BSCS NO MUNICÍPIO DE ESPERANÇA - PB
}

\section{DIVERSITY OF SPECIES AND VARIETIES OF CREOLE SEEDS IN THE CSBS IN THE MUNICIPALITY OF ESPERANÇA-PB}

\author{
Tharine da Silva Santos ${ }^{1}$; Isabella Oliveira de Araújo ${ }^{2}$; Emanoel Dias Silva ${ }^{3}$; Daniel Ferreira \\ Silva ${ }^{4}$;Maria Christine Werba Saldanha ${ }^{5}$
}

DOI: https://doi.org/10.31692/978-65-991061-7-0.1-13

\section{RESUMO}

As sementes crioulas, conhecidas no Território da Borborema-PB como Sementes da Paixão, são aquelas que, ao longo dos séculos foram desenvolvidas e vêm sendo manejadas por agricultores familiares, e outros povos tradicionais, integrando desta forma o patrimônio de diversos povos que ao longo dos tempos vêm conservando, resgatando, selecionando e valorizando variedades e espécies vegetais, mantendo a agrobiodiversidade adaptada a cada região. Essas sementes por sua vez, são armazenadas em Bancos de Sementes Familiares (BSF) e Bancos de Sementes Comunitários (BSC) pelas comunidades agrícolas. O objetivo desse trabalho é analisar o estoque dos 04 BSC do município de Esperança, Território da Borborema-PB. Caracteriza-se por sua natureza como pesquisa quantitativa realizada através de um estudo de caso em 4 (quatro) Bancos Comunitários de Sementes da cidade de Esperança-PB: Banco Municipal, Benefício, Cícero Romana e Pintado. Para o levantamento dos dados, utilizamos o sistema de monitoramento dos BSC (SALDANHA, SILVA, SANTOS, 2017), e a coleta de dados foi realizada através de visitações aos bancos e levantamento de dados junto aos gestores, associados e membros das associações envolvidas com o monitoramento dos BSC. Os quatro BCS do município de Esperança-PB (Banco Municipal, Benefício, Cícero Romana e Pintado) possuem 72 associados sendo 56 mulheres (78\%) e 16 homens (22\%) e, contabilizaram em $2017612 \mathrm{~kg}$ de sementes, compreendendo 4 espécies e 21 variedades, assim distribuídas: Feijão de Arranque (369 kg; 60,29\% e, 10 variedades, sendo carioca $(162,0 \mathrm{~kg})$, carioquinha de cacho $(60 \mathrm{~kg})$, faveta $(13 \mathrm{~kg})$, fava (13 $\mathrm{kg})$, gordo $(20 \mathrm{~kg})$, gurgutuba $(7 \mathrm{~kg})$, ovo de rolinha $(10 \mathrm{~kg})$, preto $(56 \mathrm{~kg})$, preto berabinha (4 $\mathrm{kg}$ ) e rosinha (24 kg); Feijão Macassar ou Feijão de Corda (36 kg; 4,88\% em 03 variedades: bico de ouro (2 kg), cariri (16 kg) e sempre verde $(18 \mathrm{~kg})$; Fava $(23 \mathrm{~kg} ; 3,76 \%$ e 06 variedades: branca $(2 \mathrm{~kg})$, cara larga de rama (4 kg), cara larga de moita $(1 \mathrm{~kg})$, eucalipto (8 $\mathrm{kg})$, orelha de vó $(4 \mathrm{~kg})$ e roxa $(4 \mathrm{~kg})$ e; Milho $(184 \mathrm{~kg}, 30,07 \%$ e duas variedades: jabatão amarelo $(124 \mathrm{~kg})$ e pontinha $(60 \mathrm{~kg})$. O monitoramento contínuo dos BSC permite melhor tomada de decisão pelos agricultores e instituições envolvidas relacionadas a gestão dos estoques e, subsidiar a formulação de políticas públicas adequadas ao desenvolvimento sustentável da agricultura familiar.

Palavras-Chave: Sementes Crioulas, Bancos de Sementes Comunitárias, Monitoramento, Agricultura Familiar, Agroecologia.

\section{ABSTRACT}

Creole seeds, known in the Territory of Borborema-PB as Seeds of Passion, are those that,

\footnotetext{
${ }^{1}$ Graduação Engenharia de Produção, Bolsista PROBEX - UFPB, isaoliveiraaraujo@ gmail.com

${ }^{2}$ Graduação Engenharia de Produção, Bolsista PROBEX - UFPB, tharine_silva@ hotmail.com

${ }^{3}$ Assessor Técnico da ASPTA-PB, emanoel@ aspta.org.br

${ }^{4}$ Bacharelado em Agroecologia- Bolsista PIBIC-CNPq- UFPB, daniel09ufpb@gmail.com

${ }^{5}$ Dr $^{\mathrm{a}}$ Engenharia de Produção, DEP/PPGEP/UFPB, cwerbasaldanha@gmai.com
} 
over the centuries, have been developed and are being managed by family farmers, and other traditional peoples, thus integrating the patrimony of several peoples that along of the times have been conserving, rescuing, selecting and valuing plant varieties and species, keeping agrobiodiversity adapted to each region. These seeds are in turn stored in Family Seed Banks (FSB) and Community Seed Banks (CSB) by farming communities. The objective of this work is to analyze the stock of the $04 \mathrm{CSB}$ of the municipality of Esperança, Borborema-PB Territory. It is characterized by its nature as a quantitative research carried out through a case study in 4 (four) Community Seed Banks of the city of Esperança-PB: Municipal Bank, Benefit, Cicero Romana and Pintado. In order to collect data, we used the CSB monitoring system (SALDANHA, SILVA, SANTOS, 2017), and data collection was performed through bank visits and data collection with managers, associates and members of associations involved with monitoring of CSBs. The four CSB in the municipality of Esperança-PB (Municipal Bank, Benefício, Cícero Romana and Pintado) have 72 associates, 56 women (78\%) and 16 men (22\%). In 2017 they had $612 \mathrm{~kg}$ of seeds, comprising 4 species and 21 varieties, distributed as follows: Starter Bean $(369 \mathrm{~kg}, 60.29 \%$ and 10 varieties, being carioca $(162.0 \mathrm{~kg})$, carioquinha de bunch $(60 \mathrm{~kg})$, faveta $(13 \mathrm{~kg})$, fava $(20 \mathrm{~kg})$, gurgutuba $(7 \mathrm{~kg})$, rolled egg $(10 \mathrm{~kg})$, black $(56 \mathrm{~kg})$, black bell pepper $(4 \mathrm{~kg})$ and rosinha $(24 \mathrm{~kg})$ Macassar Beans or String Beans $(23 \mathrm{~kg}, 3.76 \%$, and 06 varieties: white $(2 \mathrm{~kg}))$, and the following species: $(1 \mathrm{~kg})$, eucalyptus $(8 \mathrm{~kg})$, ear of vines $(4 \mathrm{~kg})$ and purple $(4 \mathrm{~kg}))$; Maize $(184 \mathrm{~kg}$, $30.07 \%)$ two varieties: yellow jabatão $(124 \mathrm{~kg})$ and pontinha $(60 \mathrm{~kg})$. Continuous monitoring of BSC allows better decision-making by farmers and in institutions involved in inventory management, and to support the formulation of adequate public policies for the sustainable development of family agriculture.

Keywords: Family Agriculture, Agroecology, Creoles Seeds, Community Seed Banks, Monitoring

\section{INTRODUÇÃO}

A agricultura familiar de base ecológica se adequa ao modelo de sustentabilidade, uma vez que reúne um conjunto de métodos e técnicas que vem sendo repassado através das gerações e, que visam reduzir a dependência de energia externa e o impacto ambiental, valorizando os agricultores, seus conhecimentos, suas bases sociais, seu trabalho e sua cultura e, resultando em produtos de melhor qualidade, Neste sentido, é importante que ocorra a utilização sustentável dos recursos e divisão dos benefícios da biodiversidade capazes de viabilizar as práticas tradicionais de uso dos recursos naturais e, os processos adequados de inovação e difusão tecnológica.

O Território da Borborema-PB, situado na região semi-árida da Paraíba, apresenta contrastes ambientais que ocorrem em forma de ciclos de estiagem. O modelo de produção, que vem sendo praticado pelos agricultores familiares neste Território, com apoio técnico de várias instituições, tem buscado implementar alternativas com bases agroecologias associando desenvolvimento e sustentabilidade, através de atividades economicamente viáveis, envolvendo mão de obra familiar, evitando a contaminação do meio ambiente e produzindo alimentos saudáveis e diversificados. 
Os bancos de sementes comunitários (BSC) são locais utilizados para o armazenamento de estoques de diferentes espécies e variedades de sementes crioulas que são compartilhados por famílias agricultoras. Estes bancos têm por objetivo conservar a multiplicidade de sementes, valorizar a prática de solidariedade entre as famílias, como também lutar contra às políticas públicas, que distribuem sementes sem nenhuma interação com as estratégias dos estoques das famílias nos bancos de sementes, nem com as características locais.

Segundo Oliveira (et al, 2018), os BSC constituem-se em forma segura de guardar as sementes para plantio nos próximos anos e, no momento adequado, evitando, inclusive, o risco de uso destes estoques como alimento, quando estão estocadas nos BSF (Bancos de Sementes Familiares), em função da necessidade de sobrevivência. Além disso, segundo os autores, os estoques coletivos têm aumentado a coesão social das famílias e comunidades rurais, constituindo-se em mecanismos de politica publica fundamental para o enfrentamento dos efeitos negativos da insegurança alimentar.

A Paraíba se destaca quanto ao resgate, seleção, conservação e multiplicação das sementes da paixão por meio da manutenção dos bancos de sementes familiares (BSF) e comunitários (BSC) (PETERSEN, et al, 2013). Segundo Oliveira (et al, 2018), as estratégia utilizadas pela Rede de Bancos Comunitários de Sementes, estão relacionadas à manutenção e conservação da biodiversidade existente no Território da Borborema-PB, mesmo diante dos acentuados anos seguidos de seca, possibilitado, inclusive, o resgate de variedades perdidas em determinadas comunidades. Na dinâmica de funcionamento dos BSCs, ocorre o empréstimo de volumes de sementes aos associados, que deverão ser restituídos após a colheita com o acréscimo de pequena porcentagem, a fim de que o capital coletivo seja conservado e ampliado, servindo assim de repositórios estratégicos para a conservação de variedades locais.

No monitoramento dos BSC realizado em 2017 nas 12 cidades do Território da Borborema (Alagoa Nova, Arara, Areial, Casserengue, Esperança, Lagoa de Roça, Lagoa Seca, Massaranduba, Montadas, Queimadas, Remígio, Solânea) foram identificados 63 BSC que armazenam cerca de 17 toneladas de sementes crioulas distribuídas em 10 diferentes espécies e 123 variedades.

Este artigo tem por objetivo analisar a diversidade de espécies e variedades de sementes crioulas estocadas nos BSC do município de Esperança, Território da BorboremaParaíba-Brasil, as potencialidades e ameaças deste patrimônio genético-cultural. 


\section{FUNDAMENTAÇÃO TEÓRICA}

O emprego das sementes crioulas, segundo Petersen et al (2013), é condição determinante para a aplicação da perspectiva agroecológica no manejo dos agroecossistemas, defesa e promoção da agrobiodiversidade. Estas espécies, segundo Petersen et al (2013), são portadoras de alta variabilidade genética, ou seja de características biológicas, que lhes proporciona maior plasticidade ecológica, isto é, maior resistência horizontal aos estresses ambientais. As variedades crioulas são, segundo Petersen et al (2013), portadoras de mensagens genéticas e de mensagens culturais, pois, além da pressão de seleção natural a que estão sujeitas todas as formas de vida, estas espécies e variedades foram historicamente submetidas à seleção cultural, resultando na ampla diversificação biológica, que constitui a agrobiodiversidade, um patrimônio genético-cultural reconhecido como bem comum da Humanidade.

Segundo Almeida e Cordeiro (2002, apud, Petersen et al, 2013), a “compreensão das estratégias locais de conservação e uso da agrobiodiversidade revelou a existência de sofisticados mecanismos que articulam práticas individuais e coletivas em um sistema de seguridade de sementes". Este sistem está fundamentado no armazenamento de sementes para o plantio nos anos seguintes, enriquecido pela rotina de troca de sementes entre famílias, de modo que os materiais genéticos, juntamente com os conhecimentos relacionados às qualidades intrínsecas a cada variedade local, circulam livremente nas comunidades.

\section{METODOLOGIA}

Caracteriza-se por sua natureza como pesquisa quantitativa realizada através de um estudo de caso em 4 (quatro) Bancos Comunitários de Sementes da cidade de Esperança-PB: Banco Municipal, Benefício, Cícero Romana e Pintado.

Para o levantamento dos dados, utilizamos o sistema de monitoramento dos BSC (SALDANHA, SILVA, SANTOS, 2017). No final de 2017 e início de 2018 realizou-se o levantamento dos dados em visitas realizadas a cada banco, preenchimento das planilhas relacionadas a movimentação (entrada e saída) dos estoques das espécies (feijão de arranque, feijão macassar, milho e fava) e variedades das sementes. Considerou-se os seguintes aspectos: uso pelos associados, intercâmbio entre BCS, campos de multiplicação, roçados comunitários, comercialização de sementes e doações. Também foram coletadas informações referentes ao ano de fundação, número de famílias atendidas e de associados, capacidade de estoque, formas de conservação e armazenamento, microclima e georreferenciamento, entre outros. Os dados foram obtidos junto aos gestores e associados dos BCS e, aos técnicos dos 
órgãos de apoio ao gerenciamento e monitoramento das Sementes da Paixão do Território da Borborema-PB e, no banco de dados destas entidades.

A partir dos dados coletados, realizou-se a tabulação, gerando-se gráficos e tabelas utilizando o recurso das cores como estratégia para facilitar o entendimento pelos gestores, associados e demais envolvidos. Foram utilizados tons de marrons para as variedades do feijão de arranque, rosas para as variedades do feijão macassar, verdes para as variedades do milho, azuis para as da fava e roxos para as hortaliças. Os resultados foram restituídos e validados em reuniões junto aos gestores e associados dos BCS e entidades parceiras.

\section{RESULTADOS E DISCUSSÃO}

O município de Esperança-PB está localizado no Território da Borborema-PB (Figura 1), situado aproximadamente a $159 \mathrm{~km}$ da capital João Pessoa. O município possui uma área de $163,78 \mathrm{Km}^{2}$ e 31.095 habitantes, sendo 69,56\% (21.631) residente na área urbana e 30,44\% (9.464) na área rural. A renda per capita do município em 2010 foi de $\mathrm{R} \$ 251,41$ e, o índice de desenvolvimento humano (IDH) de 0,623, classificado como médio (IBGE, 2010). Segundo dados do Censo Agropecuário 2010, constam no município 1.775 estabelecimentos de agricultura familiar nos quais, 5.171 pessoas, $16,63 \%$ da população rural, desenvolvem e sobrevivem desta atividade (Caderno territorial, 2010).

O primeiro Banco Comunitário de Sementes de Esperança foi fundado em 2000, o banco Pintado. Atualmente, o município conta com 4 BSCs em funcionamento: Banco Municipal, Beneficio, Cícero Romana e Pintado (Figuras 1 e 2). Os 4 bancos juntos dispõem de 612,00 kg de sementes, distribuídos em 4 espécies e 21 variedades. Possuem 72 associados sendo 56 mulheres $(78 \%)$ e 16 homens $(22 \%)$. 
Figura 1 - Localização do município Esperança no Território da Borborema - Paraíba - Brasil.

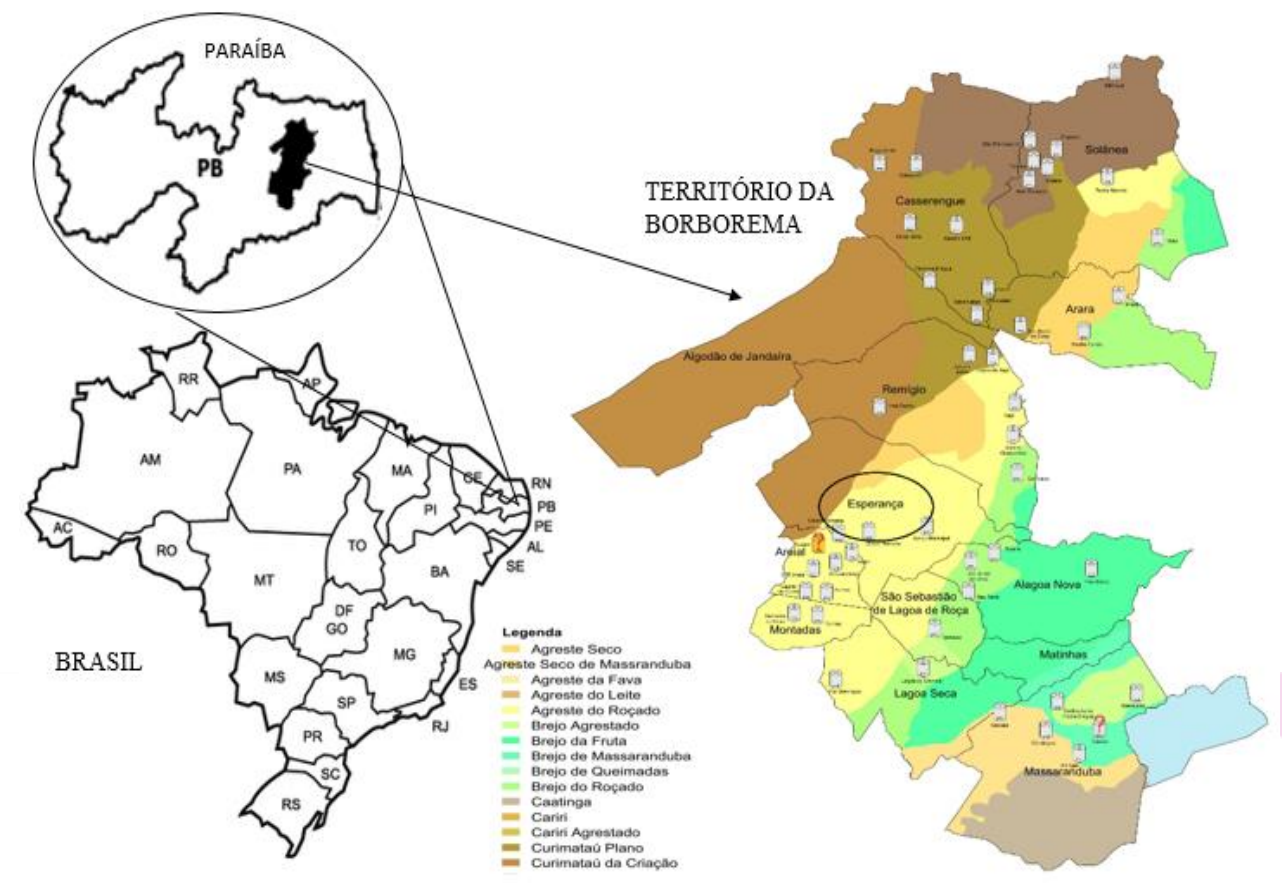

Figura 2: BSCs Municipal, Benefício, Cícero Romana e Pintado. Fonte: Próprio

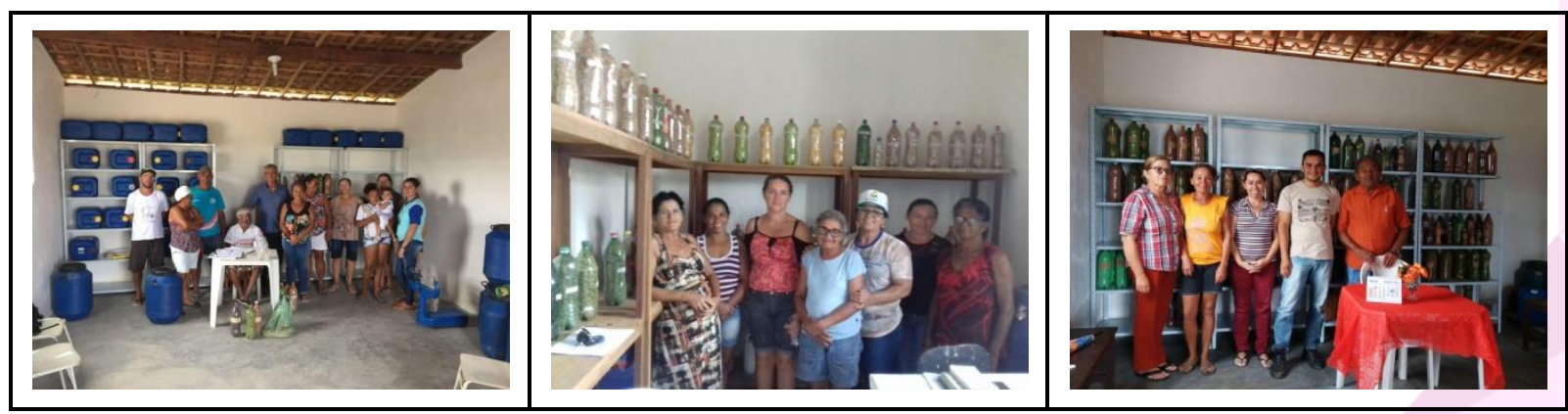

Banco Comunitário de Sementes Municipal

O Banco Municipal foi fundado em 2011, localiza-se no microclima agreste da fava e atende 7 famílias agricultoras e, 6 associados sendo, 3 homens e 3 mulheres. No ano de 2017 contou com $122 \mathrm{~kg}$ de sementes estocadas o que corresponde a quase $20 \%$ do montante da cidade, distribuídas entre 3 espécies (feijão de arranque, milho e fava) e 5 variedades.

Do total de sementes armazenadas, $88 \mathrm{~kg}$ são da espécie feijão de arranque $(72,13 \%)$ distribuídas em 2 variedades: feijão carioquinha de cacho e feijão preto. Apenas uma variedade de milho, jabatão amarelo com estoque de $30 \mathrm{~kg}(24,5 \%)$ e. 2 variedades de fava $(4,0 \mathrm{~kg}, 3,25 \%) .:$ fava de cara larga de rama e fava orelha de vó.

A espécie com mais expressiva foi o feijão, mais especificamente. a variedade feijão carioquinha (60 kg, 68,18\%). Os menores estoques são as variedades de fava, ambas as contabilizando (4 kg, 3,2\%), (Gráfico 1). 
Gráfico 1 - Variedades de sementes do BSC Banco Municipal - Esperança-PB. Fonte: Próprio

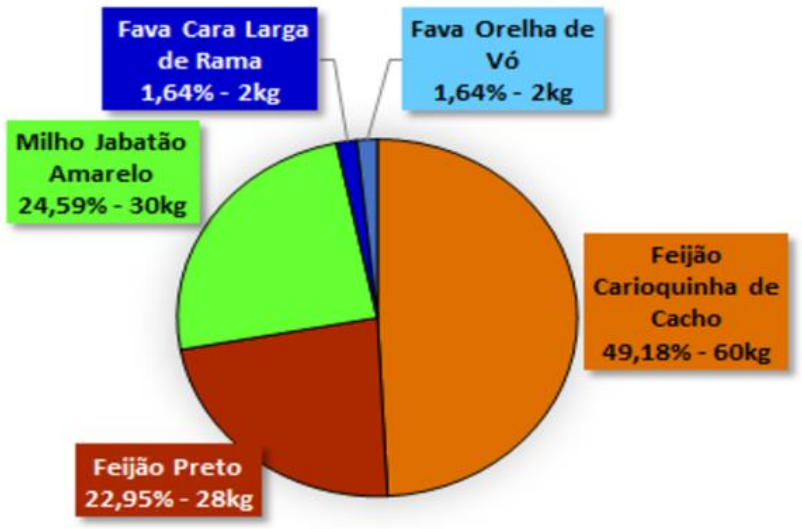

Banco Comunitário de Sementes Benefício

A banco de benefício, localizado no microclima Agreste das Favas, possui 20 associados sendo, 4 homens e 16 mulheres e, no ano de 2017 possuía $290 \mathrm{~kg}$ de sementes armazenadas, que correspondem a 47\% do estoque do município. O banco possui 4 espécies (feijão de arranque, feijão macassar, milho e fava) distribuídas em 10 variedades.

Do montante armazenado, $150 \mathrm{Kg}(51,72 \%)$ é da espécie feijão de arranque, $14 \mathrm{Kg}$ $(4,83 \%)$ feijão macassar, $120 \mathrm{Kg}(41,38 \%)$ de milho e, $6 \mathrm{Kg}$ (2,07\%) de fava. Configura uma maior armazenagem das espécies de feijão de arranque e milho totalizando 93,1\% (Gráfico 2).

Gráfico 2 - Variedades de sementes do BSC Benefício, Esperança-PB. Fonte: Próprio

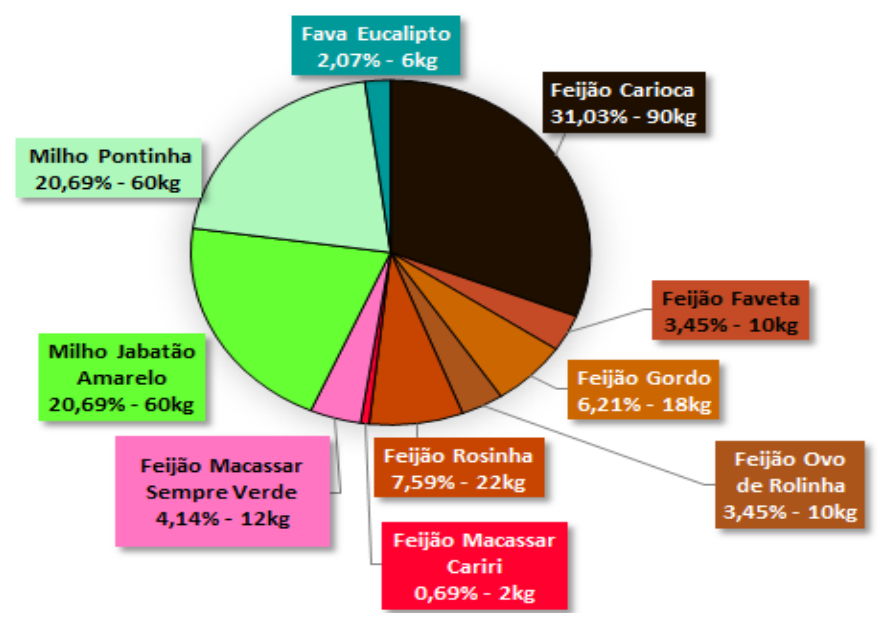

\section{Banco Comunitário de sementes Cícero Romana}

O banco comunitário de Cícero Romana, localiza-se na região do microclima Agreste da Fava, foi fundado em 2016, atende 54 famílias, possui 26 associados, entre eles 5 homens e 21 mulheres. Possui estoque de $89,0 \mathrm{~kg}$ de sementes que compreende a $14,54 \%$ do total de sementes armazenadas no município de Esperança, que estão distribuídas em 4 espécies (feijão de arranque, feijão macassar, milho e fava) e 10 variedades (feijão carioca, feijão 
faveta, feijão fava, feijão preto, feijão macassar sempre verde, milho jabatão amarelo, fava cara larga de rama, fava cara larga de moita, fava eucalipto e fava orelha de vó) (Gráfico 3).

Gráfico 3 - Variedades de sementes do BSC Cícero Romana, Esperança-PB Fonte: Próprio

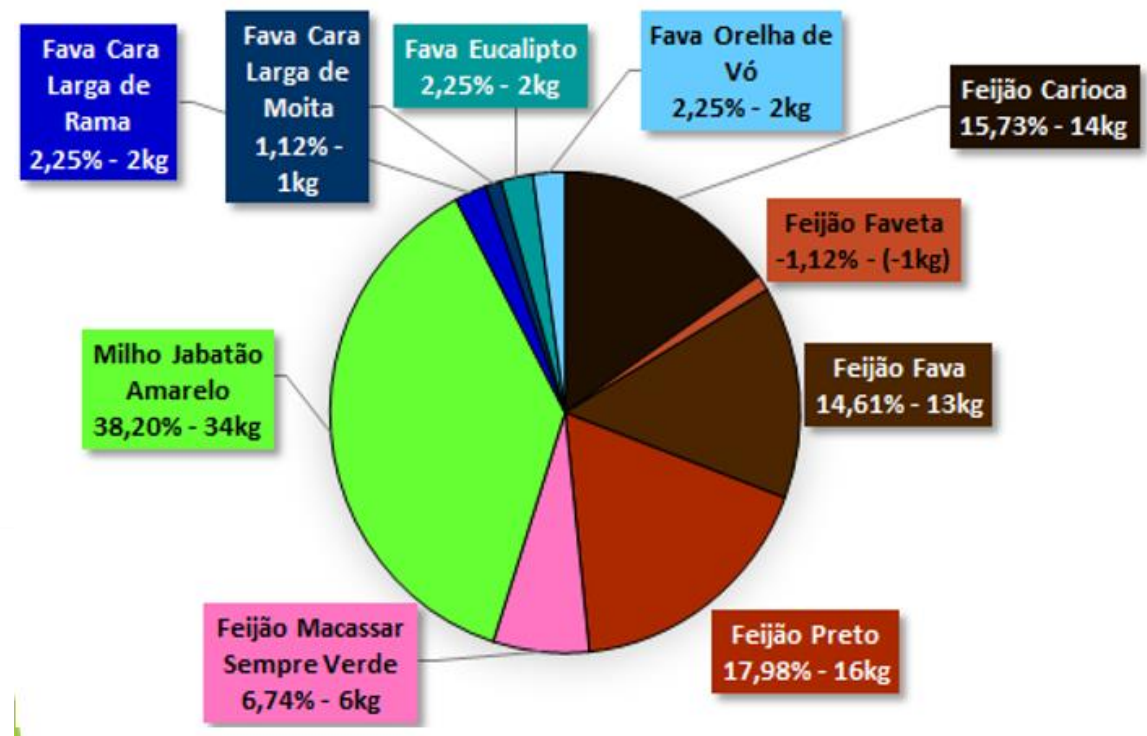

\section{Banco Comunitário de Sementes Pintado}

Fundado no ano de 2000, é o bancos mais antigo de Esperança e, contempla 18,14\% das sementes armazenadas no município, $111 \mathrm{Kg}$. Possui 20 associados sendo 4 homens e 16 mulheres. Quanto às espécies, o banco possui 3 tipos (feijão de arranque, feijão macassar e fava) subdividida em 11 variedades (feijão carioca, feijão faveta, feijão gordo, feijão gurgutuba, feijão preto, feijão preto berabinha, feijão rosinha, feijão macassar bico de ouro, feijão macassar cariri, fava branca e fava roxa). Desse total armazenado $81 \mathrm{Kg}(80,18 \%)$ é da espécie do feijão de arranque, $16 \mathrm{Kg}(14,41 \%)$ da espécie feijão macassar e $6 \mathrm{Kg}$ da espécie fava com 5,4\% do total armazenado. (Gráfico 4)

Gráfico 4 - Variedades de sementes do BSC Pintado -, Esperança-PB. Fonte: Próprio

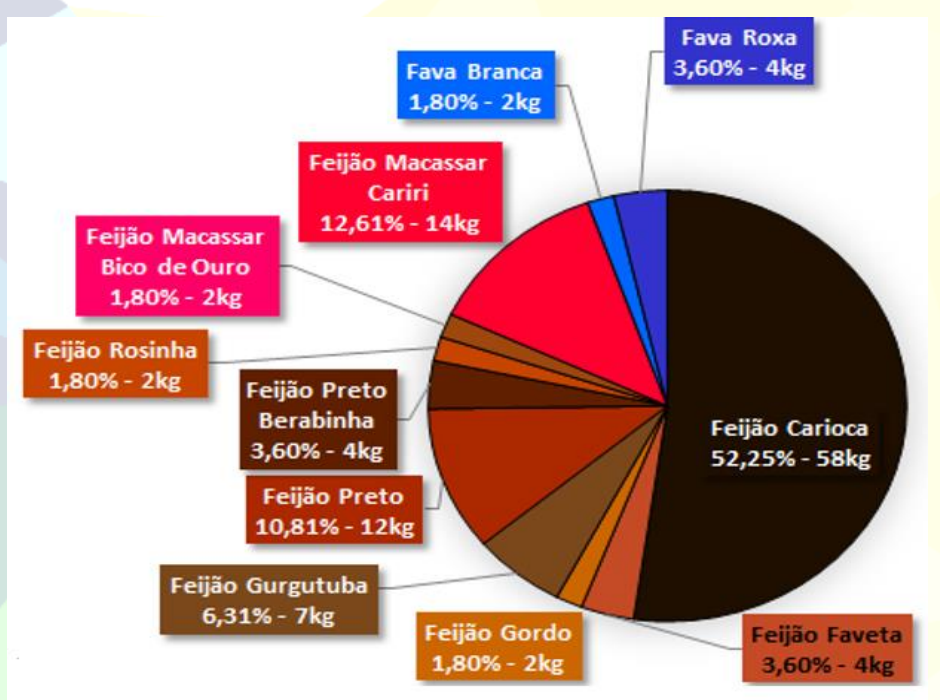

[8] 
Diversidade de Sementes Crioulas dos BSCs do município de Esperança-PB

$\mathrm{O}$ estoque de sementes dos quatro BSCs do município de Esperança-PB (Banco Municipal, Benefício, Cícero Romana e Pintado) em 2017 foi de 612 kg, compreendendo 4 espécies e 21 variedades, assim distribuídas: (Gráficos 5 e 6, Tabela 1)

Gráfico 5 - Espécies de sementes dos BSCs do município Esperança-PB. Fonte: Próprio

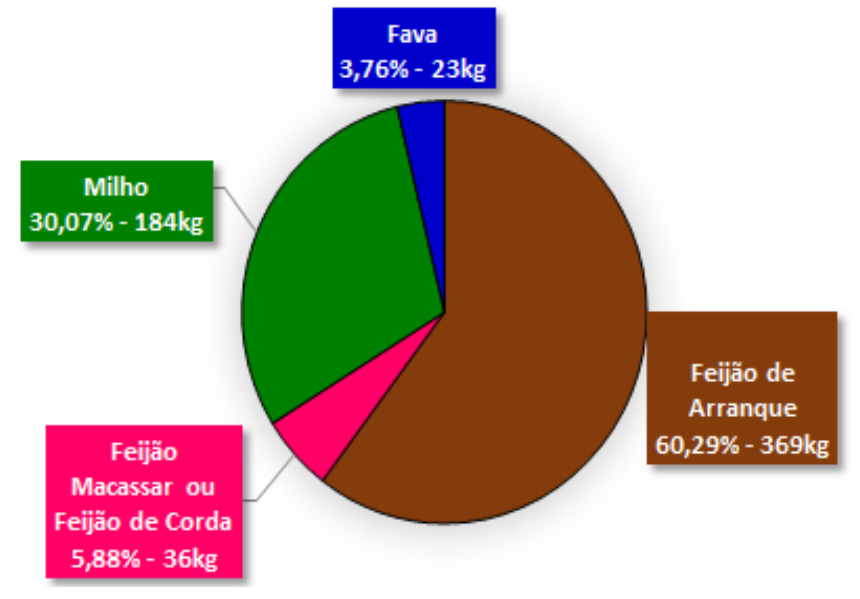

De acordo com gráfico 5, observamos que a espécie que mais predomina em Esperança é o feijão de arranque, com $369,00 \mathrm{~kg}$ e representando 60,29\% do estoque do município, contando com 10 variedades. Em segundo lugar, está o milho, tendo um total de $184 \mathrm{~kg}$, correspondendo a 30,07\% do estoque, sendo constituída por 2 variedades. O feijão macassar ou feijão de corda possui $36 \mathrm{~kg}$, em 3 variedades, representando 5,88\% do total armazenado. E por fim, a espécie que possui menor quantidade estocada é a fava, $23 \mathrm{~kg}$ e $3,76 \%$ do estoque, no entanto, é a segunda maior em número de variedades, 6. (Gráfico 6)

Gráfico 6 - Variedades de sementes Crioulas dos BSCs do município Esperança-PB. Fonte: Próprio

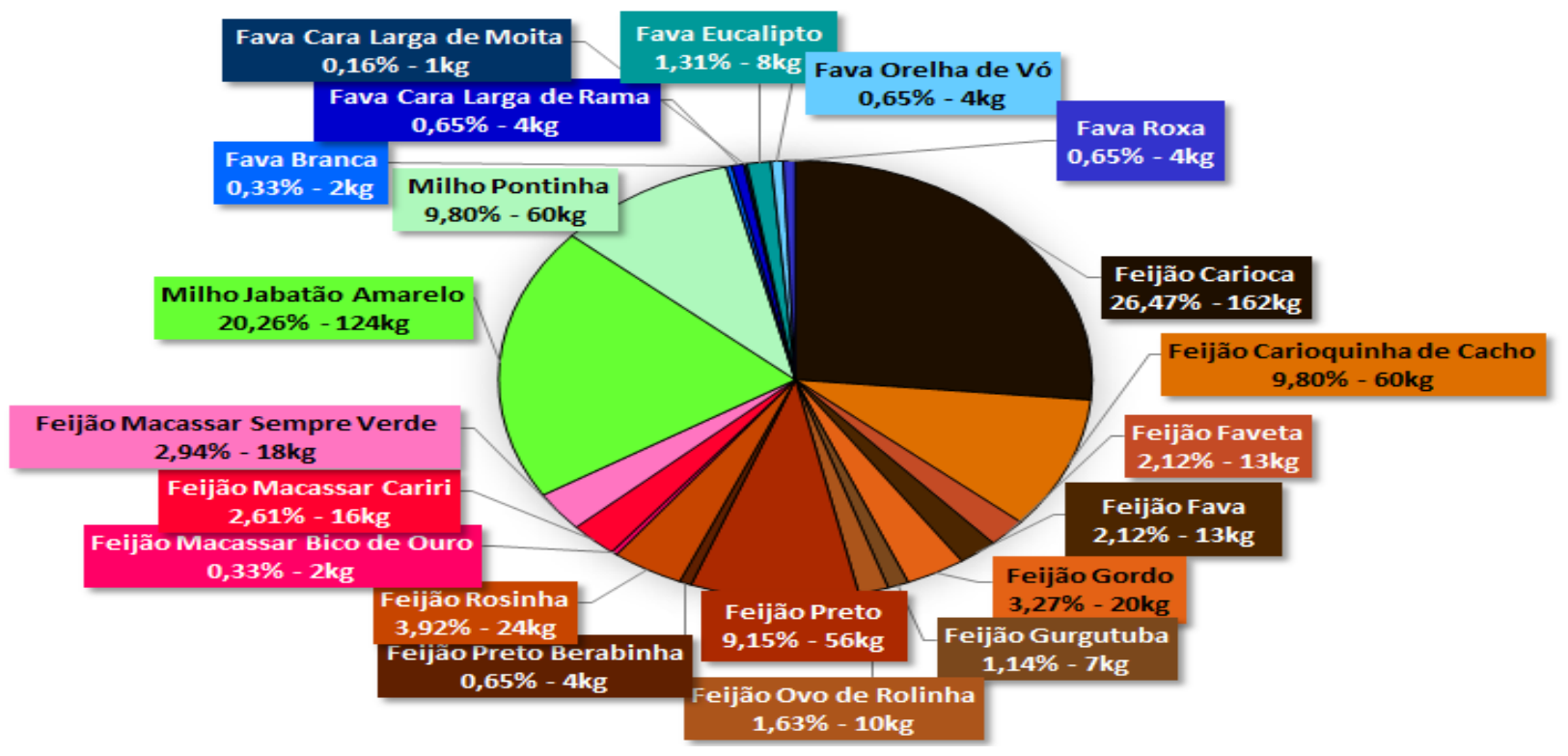


DIVERSIDADE DE ESPÉCIES E VARIEDADES DE SEMENTES CRIOULAS NOS BSCs

Tabela 1 - Estoque dos BSC do Município Esperança-PB - Ano Base: 2017. Fonte: Próprio

\begin{tabular}{|c|c|c|c|c|c|c|c|}
\hline \multicolumn{8}{|c|}{ ESTOQUE DOS BSC - MUNICÍPIO ESPERANÇA - 2017} \\
\hline \multirow{2}{*}{ Espécies } & \multirow{2}{*}{ Variedades } & \multicolumn{4}{|c|}{ Estoque BSC (kg) } & \multicolumn{2}{|c|}{$\begin{array}{l}\text { Estoque } \\
\text { Total }\end{array}$} \\
\hline & & $\begin{array}{l}\text { Banco } \\
\text { Municipal }\end{array}$ & Benefício & $\begin{array}{l}\text { Cícero } \\
\text { Romana }\end{array}$ & Pintado & $\mathbf{K g}$ & $\%$ \\
\hline \multirow{11}{*}{ Feijão de Arranque } & Carioca & & 90,00 & 14,00 & 58,00 & 162,00 & 26,47 \\
\hline & $\begin{array}{l}\text { Carioquinha de } \\
\text { Cacho }\end{array}$ & 60,00 & & & & 60,00 & 9,80 \\
\hline & Faveta & & 10,00 & $-1,00$ & 4,00 & 13,00 & 2,12 \\
\hline & Fava & & & 13,00 & & 13,00 & 2,12 \\
\hline & Gordo & & 18,00 & & 2,00 & 20,00 & 3,27 \\
\hline & Gurgutuba & & & & 7,00 & 7,00 & 1,14 \\
\hline & Ovo de Rolinha & & 10,00 & & & 10,00 & 1,63 \\
\hline & Preto & 28,00 & & 16,00 & 12,00 & 56,00 & 9,15 \\
\hline & Preto Berabinha & & & & 4,00 & 4,00 & 0,65 \\
\hline & Rosinha & & 22,00 & & 2,00 & 24,00 & 3,92 \\
\hline & $\begin{array}{ll}\text { Total: } & 10 \\
\text { variedades } & \end{array}$ & \begin{tabular}{|l|}
$\mathbf{8 8 , 0 0}$ \\
$23,85 \%$
\end{tabular} & $\begin{array}{l}\mathbf{1 5 0 , 0 0} \\
40,65 \%\end{array}$ & $\begin{array}{l}\mathbf{4 2 , 0 0} \\
11,38 \%\end{array}$ & \begin{tabular}{|l|}
89,00 \\
$24,12 \%$
\end{tabular} & 369,00 & 60,29 \\
\hline \multirow{4}{*}{$\begin{array}{l}\text { Feijão Macassar ou } \\
\text { Feijão de Corda }\end{array}$} & Bico de Ouro & & & & 2,00 & 2,00 & 0,33 \\
\hline & Cariri & & 2,00 & & 14,00 & 16,00 & 2,61 \\
\hline & Sempre Verde & & 12,00 & 6,00 & 0,00 & 18,00 & 2,94 \\
\hline & $\begin{array}{ll}\text { Total: } & \mathbf{3} \\
\text { variedades } & \end{array}$ & 0,00 & \begin{tabular}{|l|}
14,00 \\
$38,89 \%$
\end{tabular} & $\begin{array}{l}\mathbf{6 , 0 0} \\
16,67 \%\end{array}$ & \begin{tabular}{|l|}
$\mathbf{1 6 , 0 0}$ \\
$44,44 \%$
\end{tabular} & 36,00 & 5,88 \\
\hline \multirow{3}{*}{ Milho } & Jabatão Amarelo & 30,00 & 60,00 & 34,00 & & 124,00 & 20,26 \\
\hline & Pontinha & & 60,00 & & & 60,00 & 9,80 \\
\hline & $\begin{array}{ll}\text { Total: } & 2 \\
\text { variedades } & \end{array}$ & \begin{tabular}{|l|}
$\mathbf{3 0 , 0 0}$ \\
$16,30 \%$
\end{tabular} & $\begin{array}{l}\mathbf{1 2 0 , 0 0} \\
65,22 \%\end{array}$ & $\begin{array}{l}\mathbf{3 4 , 0 0} \\
18,48 \%\end{array}$ & 0,00 & 184,00 & 30,07 \\
\hline \multirow{7}{*}{ Fava } & Branca & & & & 2,00 & 2,00 & 0,33 \\
\hline & $\begin{array}{l}\text { Cara Larga de } \\
\text { Rama }\end{array}$ & 2,00 & & 2,00 & & 4,00 & 0,65 \\
\hline & $\begin{array}{l}\text { Cara Larga de } \\
\text { Moita }\end{array}$ & & & 1,00 & & 1,00 & 0,16 \\
\hline & Eucalipto & & 6,00 & 2,00 & & 8,00 & 1,31 \\
\hline & Orelha de Vó & 2,00 & & 2,00 & & 4,00 & 0,65 \\
\hline & Roxa & & & & 4,00 & 4,00 & 0,65 \\
\hline & Total: & 4,00 & 6,00 & 7,00 & 6,00 & 23,00 & 3,76 \\
\hline
\end{tabular}




\begin{tabular}{|l|l|l|l|l|l|l|l|}
\hline & variedades & $17,39 \%$ & $26,08 \%$ & $30,43 \%$ & $26,08 \%$ & & \\
\hline Totais & $\mathbf{2 1}$ & $\begin{array}{l}\mathbf{1 2 2 , 0 0} \\
19,93 \%\end{array}$ & $\begin{array}{l}\mathbf{2 9 0 , 0 0} \\
47,38 \%\end{array}$ & $\begin{array}{l}\mathbf{8 9 , 0 0} \\
14,54 \%\end{array}$ & $\begin{array}{l}\mathbf{1 1 1 , 0 0} \\
18,14 \%\end{array}$ & $\mathbf{6 1 2 , 0 0}$ & $\mathbf{1 0 0}$ \\
\hline
\end{tabular}

Dentre as 21 variedades de sementes da paixão estocadas nos BSC em Esperança, exceto o banco municipal, o feijão carioca possui o maior estoque, $162 \mathrm{~kg}$, representando 26,47\%. Representando segundo lugar, temos o milho jabatão amarelo, com $124 \mathrm{~kg}$, representando 20,26\% do estoque. Logo após temos mais três variedades que se destacam, feijão carioquinha de cacho e milho pontinha, cada um possui $60 \mathrm{~kg},(9,8 \%)$ do estoque e, o feijão preto com $56 \mathrm{~kg}(9,15 \%)$. Essas espécies anteriormente citadas, totalizam $462 \mathrm{~kg}$ de sementes, correspondendo a 75,48\% do estoque de Esperança - PB.

Ao analisar o gráfico 6 , observamos que nenhuma variedade está presente no estoque dos quatro BSC. Três variedades estão presentes em três BSC: feijão carioca (162 kg), feijão preto $(56 \mathrm{~kg})$ e milho jabatão amarelo $(124 \mathrm{~kg})$.

Atenção especial deve ser dada às variedades existentes em um único BCS e que possuem estoques igual ou inferior à $2,0 \mathrm{~kg}$, considerados estoques críticos: feijão macassar bico de ouro (2 kg), fava branca (2 kg) e fava cara larga de moita (1 kg).

Verifica-se um aumento de 244\% no estoque entre $2016(178 \mathrm{~kg})$ e $2017(612 \mathrm{~kg})$, com aumento de estoque em todos os bancos, exceto o banco municipal. O BSC Benefício não possuía estoque em 2016, e em 2017 foram resgatadas as seguintes espécies e variedades: - Feijão de arranque: carioca $(90 \mathrm{~kg})$, faveta $(10 \mathrm{~kg})$, gordo $(18 \mathrm{~kg})$, ovo de rolinha $(10 \mathrm{~kg}) \mathrm{e}$ rosinha (22 kg); - Feijão macassar: cariri (2 kg) e sempre verde (12 kg); milho (600 kg) e pontinha (6 kg) e; - Fava: eucalipto $(6 \mathrm{~kg})$, totalizando $296 \mathrm{~kg}$ de sementes estocadas.

As variedades que permaneceram com o mesmo estoque de 2016 para 2017, foram: carioquinha de cacho $(60 \mathrm{~kg})$, milho jabatão amarelo $(30 \mathrm{~kg})$, fava cara larga de rama $(2 \mathrm{~kg})$ e fava orelha de vó $(2 \mathrm{~kg})$.

\section{CONCLUSÃO}

O monitoramento dos Bancos de Sementes Comunitários tem por finalidade contribuir e evidenciar variedades de espécies de sementes crioulas existentes em um município, território ou mesmo uma região, fornecendo subsídio para avaliar forças e fraquezas com o intuito de preservar o patrimônio genético-cultural local.

De acordo com as análises dos bancos, percebeu-se que existem variedades em apenas um banco, sendo elas: feijão macassar bico de ouro, fava branca e fava cara larga da mata 
com estoques igual ou inferiores a $2 \mathrm{Kg}$, consideradas críticas para o município.

O monitoramento contínuo dos BSC, sua restituição e validação junto aos interessados, contribui positivamente na tomada de decisão e gestão de estoque das sementes de maneira que minimize o risco de perda de patrimônio genético e cultural. As estratégias podem ser encorajadas através de promoção de intercâmbio de sementes entre BSC ou BSF, comercialização e produção de campos de multiplicação para produção dos estoques críticos.

Para além das discussões entre agricultores, Aspta e sindicatos, os dados podem ser utilizados por gestores públicos como forma de embasamento para a formulação e implementação de políticas públicas que contemplem a cultura local e a preservação do patrimônio da região.

\section{REFERÊNCIA}

CENSO AGROPECUÁRIO. Rio de Janeiro: IBGE, 2010. Disponível em: <www.ibge.gov.br>. Acesso em: 10/05/2018.

CENSO DEMOGRÁfiCO. Rio de Janeiro: IBGE, 2010. Disponível em: <www.ibge.gov.br>. Acesso em: 10/05/2018.

HENDRICK, H. W.; KLEINER, B. H. Macroergonomia: uma introdução aos projetos de sistemas de trabalho. Rio de Janeiro: Virtual Científica, 2006.

LONDRES, F. Sementes da diversidade: a identidade e o futuro da agricultura familiar. Rio de Janeiro: Revista Agriculturas, 2014. V.11, n.1, p.04-08. Disponível em: $<$ http://aspta.org.br/wp-content/uploads/2014/05/Agriculturas_V11N1.pdf >. Acesso em: $22 / 06 / 2018$

MORAIS, R. C.; ARAÚJO, S. L., OLIVEIRA, P. N.; OLIVEIRA, R. N.; SANTOS, A. SILVA. Sementes da Paixão cultivando vidas e saberes no Cariri, Curimataú e Seridó paraibano. Rio de Janeiro: Revista Agriculturas, 2014. V.11, n.1, p.19-23. Disponível em: <http://aspta.org.br/wp-content/uploads/2014/05/Agriculturas_V11N1>. Acesso em: 22/06/2018.

OLIVEIRA, L. C. L.de; DIAS, E.; CURADO, F. F.; OLIVEIRA, A. E.; MUNIZ, E. L. S.; SANTOS, A. S.. Perspectivas da pesquisa e gestão dos bancos de sementes comunitários no Polo da Borborema, Paraíba. Cadernos de Agroecologia, 2018. Vol. 13, N 1, Jul. 2018, Dispon. em: http://cadernos.aba-agroecologia.org.br/index.php/cadernos/article/view/898/378. Acesso em: 22/09/2018

PETERSEN, P.; SILVEIRA, L. M.; SILVA, E. D.; SANTOS, A. S.; CURADO, F. Sementes ou grãos? Lutas para desconstrução de uma falsa dicotomia - Semente é tudo aquilo que nasce. Rio de Janeiro: Revista Agriculturas, 2013. V. 10, p. 36-45. Disponível em: aspta.org.br/wp-content/uploads/2013/06/artigo-5.pdf. Acesso em: 22/09/2018

SALDANHA M. C. W.; SILVA, E. D.; SANTOS, T. S. Sistema de Monitoramento dos BSC 
SANTOS, et al.

(Bancos de Sementes Comunitárias) do Território da Borborema-PB. Software não registado. João Pessoa: UFPB, 2017.

SALDANHA M. C. W. Ergonomia de concepção de uma plataforma Line Oriented Flight Training (LOFT) em uma companhia aérea brasileira: a relevância do processo de construção social de projeto. 236f. Tese Doutorado em Engenharia de Produção, COPPE/UFRJ, 2004. 